\title{
Hsp70:CHIP Ubiquitinates Dysfunctional but Not Native Neuronal NO Synthase
}

\author{
(D)Amanda K. Davis, Natalie F. McMyn, Miranda Lau, Yoshihiro Morishima, \\ and Yoichi Osawa
}

Department of Pharmacology, University of Michigan, Ann Arbor, Michigan

Received March 4, 2020; accepted June 11, 2020

\begin{abstract}
Heat shock protein (Hsp) 70 modulators are being developed to enhance the removal of toxic proteins in a variety of protein misfolding diseases. In the course of our studies on neuronal nitric oxide synthase (nNOS), a client of the Hsp90 and Hsp70 chaperone system, we have established that inactivation of nNOS by heme or tetrahydrobiopterin $\left(\mathrm{BH}_{4}\right)$ alteration and loss triggers ubiquitination by the Hsp70-associated E3 ligase c-terminus of Hsp70-interacting protein (CHIP) and subsequent degradation in cells. Although in cells Hsp90 and Hsp70 work together to maintain protein quality control, in this study, we specifically developed an assay to assess the selectivity of the Hsp70:CHIP complex for inactivated nNOS. We developed a highly sensitive ELISA to measure Hsp70:CHIP-dependent nNOS ubiquitination without interference from direct ubiquitination by $\mathrm{CHIP}$, as evidenced by $\mathrm{Bcl}-2$ associated athanogene 1-M completely abolishing ubiquitination. To further validate the assay we demonstrated, JG-98, a rhodocyanin compound that acts on Hsp70 but not its inactive structural analog JG258 , enhances the ubiquitination of nNOS 3-fold. Utilizing this assay, we have shown that the Hsp70:CHIP complex preferentially ubiquitinates heme-deficient nNOS (apo-nNOS)
\end{abstract}

over heme-containing nNOS (holo-nNOS). Moreover, depletion of nNOS-bound $\mathrm{BH}_{4}$ triggers ubiquitination of holo-nNOS by the Hsp70:CHIP complex. Most importantly, JG-98 was shown to enhance the ubiquitination of only dysfunctional nNOS while leaving the native functional nNOS untouched. Thus, the finding that enhancing Hsp70:CHIP-mediated ubiquitination does not affect native proteins has important pharmacological implications. Moreover, development of a facile in vitro method for Hsp70:CHIP-mediated ubiquitination will be beneficial for testing other Hsp70 modulators.

\section{SIGNIFICANCE STATEMENT}

The heat shock protein 70 (Hsp70):c-terminus of Hsp70interacting protein (CHIP) complex facilitates the ubiquitination and subsequent degradation of several hundred-client proteins, and activation of Hsp70 has been suggested as a therapeutic strategy to enhance the degradation of disease-causing proteins. The current study shows that the pharmacological activation of Hsp70 enhances the ubiquitination of dysfunctional but not native nNOS, and it suggests that this therapeutic strategy will likely be highly selective.

\section{Introduction}

The heat shock protein (Hsp) 90 and Hsp 70 chaperone system regulates the degradation of several hundred client proteins, including those implicated in cancer (Isaacs et al., 2003), neurodegenerative disease (Pratt et al., 2015), and polyglutamine disorders (Davis et al., 2020). Hsp90 and Hsp70 play a yin and yang role; Hsp90 stabilizes client proteins preventing degradation (Pratt et al., 2008), whereas Hsp70 recruits E3 ligases, such as c-terminus of Hsp70 interacting protein (CHIP), promoting ubiquitination and degradation (McDonough and Patterson, 2003). The prevailing model suggests that Hsp90 and Hsp70 surveil protein-folding clefts

This work was supported by the National Institutes of Health National Institute of General Medical Sciences [Grants RO1-GM077430 (to Y.O.) and T32-GM007767 (to A.K.D.)] and the PhRMA Foundation Predoctoral Fellowship in Pharmacology/Toxicology (to A.K.D.). The authors are all participants in The University of Michigan Medical School's Protein Folding Diseases Initiative.

https://doi.org/10.1124/mol.120.119990. and target non-native client proteins for degradation; this selectivity makes the chaperone system an attractive therapeutic target (Pratt et al., 2014). Traditionally, the chaperone system has been targeted with Hsp90 inhibitors, and although numerous Hsp90 inhibitors have entered clinical trials for treatment of cancer, none have advanced to clinical use, in part because of dose-limiting toxicity (Sanchez et al., 2020). The role of Hsp90 in the stabilization of client proteins in their native state raises concerns of on-target toxicity. Thus, interest in activation of Hsp70 has increased, especially in light of recent experiments demonstrating cochaperones and small molecules that target Hsp70 can enhance the degradation of various client proteins (Wang et al., 2013; Davis et al., 2020). It has been demonstrated that this degradation occurs through enhancing Hsp70:CHIP-dependent ubiquitination, but the selectivity of this approach for non-native client protein conformations have only been tested in a few cases.

The earliest in vitro studies on the selectivity of Hsp70:CHIPdependent ubiquitination demonstrated that heat-denatured

ABBREVIATIONS: apo-nNOS, heme-deficient nNOS; BAG1-M, Bcl-2 associated athanogene 1-M; $\mathrm{BH}_{4}$, tetrahydrobiopterin; $\mathrm{CHIP}$, c-terminus of Hsp70-interacting protein; D-NNA, NG-Nitro-D-arginine; DTT, dithiothreitol; holo-nNOD, heme-containing nNOS; HIP, Hsp70-interacting protein; HRP, horseradish peroxidase; Hsp, heat shock protein; L-NNA, $N^{G}$-Nitro-L-arginine; nNOS, neuronal nitric oxide synthase. 
firefly luciferase is readily ubiquitinated over its native form (Murata et al., 2001). However, this selectivity for denatured firefly luciferase has been ascribed to the Hsp70:CHIP and Hsp90:CHIP complex (Murata et al., 2001) as well as CHIP alone (Ren et al., 2011); therefore, it is unclear what role, if any, Hsp70 plays in ubiquitination selectivity. In the current study, we investigate the selectivity of ubiquitination by the Hsp70:CHIP complex in the absence of Hsp90 and determine the effect of pharmacological activation of Hsp70 in vitro, utilizing neuronal nitric oxide synthase (nNOS) as a model client protein. We take advantage of established methods that alter the native functional nNOS to dysfunctional nNOS forms by altering the amount of bound heme or tetrahydrobiopterin $\left(\mathrm{BH}_{4}\right)$ (Bender et al., 2000a; Dunbar et al., 2004; Kamada et al., 2005). These dysfunctional states do not allow the formation of NO but are not denatured forms, as they can still bind calmodulin and function as an NADPH oxidase (Abu-Soud et al., 1994a; Gebhart et al., 2019). It is noteworthy that for all three NOS isoforms, depletion of $\mathrm{BH}_{4}$ leads to a dysfunctional form of the enzyme that can cause oxidative stress (Gebhart et al., 2019), and $\mathrm{BH}_{4}$ depletion of $\mathrm{nNOS}$ and the endothelial isoform, endothelial nitric oxide synthase, is implicated in heart disease (Farah et al., 2018). Thus, these dysfunctional forms exist in vivo, and the ubiquitination results from our current in vitro study can be readily compared with the enhanced ubiquitination and turnover of dysfunctional nNOS in cellular and in vivo studies.

To directly assess the Hsp70:CHIP-dependent ubiquitination of nNOS in vitro, we had to overcome the major pitfall that CHIP can directly ubiquitinate proteins independent of recruitment by Hsp70 under in vitro conditions (Ren et al., 2011; Ravalin et al., 2019). We addressed this by developing a highly sensitive, quantitative, and reproducible ELISA to measure nNOS ubiquitination; this ELISA enabled the optimization of reaction stoichiometry and kinetics for Hsp70:CHIP-dependent ubiquitination, free of direct ubiquitination by CHIP. We verified the complete dependence on Hsp70 by demonstrating that the cochaperone Bcl-2 associated athanogene 1-M (BAG$1 \mathrm{M}$ ) and the small molecule JG-98 abolished and enhanced nNOS ubiquitination, respectively, as expected from their known actions on Hsp70 (Gässler et al., 2001; Li et al., 2013). To address client protein selectivity of this system, we found that the Hsp70:CHIP complex readily ubiquitinated hemedeficient apo-nNOS over heme-sufficient holo-nNOS and that depletion of substrate-binding cleft-bound $\mathrm{BH}_{4}$ triggered the ubiquitination of holo-nNOS. Moreover, treatment with JG-98 increased the ubiquitination of dysfunctional nNOS but not nNOS in its native state. These findings strongly suggest that pharmacological activation of Hsp70 will maintain the inherent selectivity of the chaperone machinery and may offer a therapeutic strategy to specifically target disease-causing client proteins for degradation.

\section{Materials and Methods}

Materials. ATP, NADP ${ }^{+}$, glucose-6-phosphate, glucose-6-phosphate-dehydrogenase, and guanabenz were purchased from SigmaAldrich (St. Louis, MO). $\mathrm{N}^{\mathrm{G}}$-Nitro-L-arginine (L-NNA) was purchased from Cayman Chemical (Ann Arbor, MI). Sodium citrate, RIPA buffer, and 1-Step Slow 3,3',5,5' -tetramethylbenzidine-ELISA substrate solution was purchased from Fisher (Hampton, NH). $\mathrm{BH}_{4}$ was purchased from Schircks Laboratories (Jona, Switzerland); $\mathrm{BH}_{4}$ was prepared in an aqueous solution containing equal molar dithiothreitol (DTT). $\mathrm{N}^{\mathrm{G}}$-Nitro-D-arginine (D-NNA) and the mono- and polyubiquitinylated conjugates monoclonal antibody clone FK2 [horseradish peroxidase (HRP) conjugate] was purchased from Enzo Life Sciences (Farmingdale, NY). DYKDDDDK-tag (FLAG) Antibody Plate (clear, $8 \times 12$ strip) was purchased from GenScript (Piscataway, NJ). Ubiquitin-activating enzyme was purchased from R\&D Systems (Minneapolis, MN). The cDNA for expressing the glutathione S-transferase-tagged UbcH5a (E2, ubiquitin carrier protein) was kindly provided by C. M. Pickart (Johns Hopkins Medical School, Baltimore, MD). cDNA for His-CHIP was kindly provided by C. Patterson (University of North Carolina, Chapel Hill). The cDNA for rat nNOS was kindly provided by Dr. Solomon Snyder (The Johns Hopkins Medical School). The cDNA for His-HA-tagged ubiquitin was from Dr. Yi Sun (University of Michigan, Ann Arbor). Human Hsp70 cDNA was kindly provided by Dr. David Toft (Mayo Clinic, Rochester, MN). Anti-nitric oxide synthase, brain (1409-1429) antibody produced in rabbit was purchased from Sigma-Aldrich, and IRDye 680RD goat anti-rabbit IgG secondary antibody was purchased from LI-COR Biosciences (Lincoln, Nebraska).

Expression and Purification of Proteins. Glutathione S-transferase-tagged UbcH5a was bacterially expressed and purified by GSH-Sepharose affinity chromatography as previously described (You and Pickart, 2001). His-CHIP and His-HA-ubiquitin were bacterially expressed and purified by nickel-nitrilotriacetic acid affinity chromatography as previously described (Ballinger et al., 1999). Murine BAG-1M was expressed in Escherichia coli and purified as previously described (Stuart et al., 1998). Hsp70 was bacterially expressed and purified by ATP-agarose chromatography adapted from Dittmar et al. (1996). Hsp90 was purified from rabbit reticulocyte lysate by sequential chromatography on DE52, hydroxylapatite, and ATP-agarose as previously described (Dittmar et al., 1996). Rat untagged and c-terminal FLAG-tagged apo-nNOS (Morishima et al., 2016) was expressed in Sf9 insect cells using a recombinant baculovirus and purified by $2^{\prime}, 5^{\prime}$-ADP-sepharose and gel-filtration chromatography as described previously (Bender et al., 1999). The same method was used for the generation of FLAG-tagged holo-nNOS, except that exogenous heme was added to Sf9 cells (Bender et al., 1999). The FLAG-tagged and untagged nNOS was ubiquitinated to the same degree by a purified protein system, as determined by Western blot analysis (data not shown). The specific activity of the FLAG-tagged and untagged nNOS were equivalent, as determined by an oxyhemoglobin assay for the measurement of nitric oxide (Morishima et al., 2016).

In Vitro Ubiquitination Reaction with Purified Proteins. A purified protein ubiquitination reaction containing purified FLAGtagged nNOS $(0.2 \mu \mathrm{M})$, Hsp70 (1.0 $\mu \mathrm{M})$, His-CHIP (1.0 $\mu \mathrm{M})$, UbcH5a (1 $\mu \mathrm{M})$, ubiquitin-activating enzyme $1(0.1 \mu \mathrm{M})$, His-HA-ubiquitin $(100 \mu \mathrm{M})$, and $\operatorname{ATP}(100 \mu \mathrm{M})$ in a total volume of $20 \mu \mathrm{l}$ of $50 \mathrm{mM}$ Hepes $\mathrm{pH} 7.4,100 \mathrm{mM} \mathrm{KCl}$, and $5 \mathrm{mM}$ DTT was incubated at $22^{\circ} \mathrm{C}$ for the specified time. Ubiquitination reactions were then analyzed by ELISA or Western blotting as described below.

ELISA for nNOS Ubiquitination. Ubiquitination reactions were diluted in RIPA buffer to a final FLAG-nNOS concentration of $0.03 \mu \mathrm{M}$, and $100 \mu \mathrm{l}$ was added per well to an anti-FLAG 96-well plate. The plate was incubated for 4 hours at $4^{\circ} \mathrm{C}$ and then washed three times with RIPA buffer and once with PBS. Wells were treated with $100-\mu l$ anti-ubiquitin antibody conjugated to HRP (1:2000). The plate was incubated for 1 hour at room temperature and then washed three times with PBS containing $1 \%$ Tween 20 and once with PBS. To measure HRP activity, wells were treated with $100 \mu \mathrm{l} 3,3^{\prime}, 5,5^{\prime}$ tetramethylbenzidine, and absorbance at $370 \mathrm{nM}$ was read every 7 seconds for 120 seconds. Raw values are shown in Fig. 2, but in all other figures on the ELISA, values were corrected for the non-nNOS-specific HRP signal, which is shown in Fig. 2, and HRP activity was reported relative to an apo-nNOS untreated control in all other studies. 
Western Blotting. Ubiquitination reactions were treated with $4 \times$ laemmli buffer and heated for 5 minutes at $100^{\circ} \mathrm{C}$. A total of $0.48 \mu \mathrm{g}$ FLAG-nNOS was loaded per well, resolved on a $5 \%$ (w/v) SDS-PAGE gel, and transferred to a polyvinylidene fluoride membrane for 2 hours at $100 \mathrm{~V}$. The blot was probed with an anti-nNOS primary antibody and a fluorescent anti-rabbit IgG secondary antibody. Higher molecular weight nNOS ubiquitin conjugates were quantified in Image Studio from LI-COR Biosciences, and values were corrected for background and reported as absorbance (arbitrary units).

Alteration of the Substrate-Binding Cleft of nNOS. nNOS $(1 \mu \mathrm{M})$ was incubated with or without an NADPH regenerating system containing NADP+ $(400 \mu \mathrm{M})$, glucose-6-phosphate $(10 \mathrm{mM})$, glucose-6phosphate-dehydrogenase $(1 \mathrm{U} / \mathrm{ml})$, sodium citrate $(10 \mu \mathrm{M})$, calmodulin $(1 \mu \mathrm{M})$, and calcium $(100 \mu \mathrm{M})$ in $50 \mathrm{mM}$ Hepes $\mathrm{pH} 7.4,100 \mathrm{mM}$ $\mathrm{KCl}$, and $5 \mathrm{mM}$ DTT for 30 minutes at $30^{\circ} \mathrm{C}$. The preincubation was then diluted 5 -fold into the purified protein in vitro ubiquitination reaction as described above.

Statistical Analysis. A biologic sample (n) represents an independent ubiquitination reaction individually treated with the appropriate variable. Statistical analysis was performed with GraphPad PRISM version 8 (San Diego, CA). Western blotting data shown in Fig. 1A was fit with a second order polynomial (quadratic) equation. ELISA data shown in Fig. 2B was fit with a simple linear regression. Data from concentration-response experiments, Figs. 3 and 5, were fit with a variable slope dose-response equation (sigmoidal fourparameter logistic curve). Statistical significance was determined using a repeated measures one-way ANOVA followed by a Dunnett's multiple comparison test for Fig. 2B, an ordinary one-way ANOVA followed by a Tukey's multiple comparison test for Fig. 4A, and an ordinary one-way ANOVA followed by a Dunnett's multiple comparisons test for Fig. 4B.

\section{Results}

Purified Protein System for the Study of Hsp70-Dependent Ubiquitination of nNOS by CHIP. We sought to establish an in vitro method to study Hsp70mediated ubiquitination of nNOS by CHIP. In previous studies, Western blotting was used to measure nNOS ubiquitination as shown in Fig. 1. Thus, based on previous studies, we incubated FLAG-tagged apo-nNOS with a purified protein mixture containing the E1 ubiquitin activating enzyme, an E2 ubiquitinconjugating enzyme, the E3-ligase CHIP, and Hsp70 for increasing amounts of time at $22^{\circ} \mathrm{C}$ and quenched the reaction with sample buffer. Sample buffer-treated reactions were then resolved by SDS-PAGE and visualized by Western blotting with an anti-nNOS antibody (Fig. 1A). Higher molecular weight bands corresponding to nNOS-ubiquitin conjugates were then quantified (Fig. 1B). Because of the large amount of
nNOS used for visualization by Western blotting, the unmodified nNOS band tended to obscure bands with one or a few ubiquitin added, favoring the measurement of higher molecular weight poly-ubiquitin bands (Fig. 1A). Additionally, there was a large error associated with the quantification of higher molecular weight poly-ubiquitin bands (Fig. 1B).

To help overcome these limitations, we developed an ELISA to measure nNOS ubiquitination (Fig. 2). In this assay, FLAGtagged apo-nNOS was incubated with the purified protein ubiquitination reaction mixture and then immobilized on an anti-FLAG 96-well plate. An anti-ubiquitin antibody conjugated to HRP, capable of detecting both mono- and polyubiquitin chains, was then used to probe for nNOS-ubiquitin conjugates and HRP activity was measured. A shown in Fig. 2A, a time course was performed with ubiquitin reaction mixtures containing FLAG-tagged (closed circles) or untagged apo-nNOS (open circles). The signal for the mixture containing FLAG-tagged apo-nNOS increased over time, whereas the signal for the mixture containing untagged apo-nNOS did not, demonstrating that the signal is dependent on FLAGtagged nNOS. To assess the dependence of the signal on CHIP-mediated ubiquitination, FLAG-tagged apo-nNOS was incubated for 15 minutes with a complete ubiquitination reaction mixture or mixtures lacking CHIP or ubiquitin (Fig. 2B). Omission of CHIP or ubiquitin significantly decreased HRP activity. These results demonstrate that the signal generated by the ELISA is representative of CHIPdependent ubiquitination of FLAG-tagged nNOS. Moving forward nNOS ubiquitination was calculated by correcting for the non-nNOS-specific signal shown in Fig. 2A and was reported as HRP activity relative to untreated apo-nNOS control.

One potential pitfall of utilizing a purified protein system to study Hsp70-mediated ubiquitination in vitro is that Hsp70independent ubiquitination by CHIP may occur (Ren et al., 2011). Simple omission of Hsp70 from the mixture does not provide an accurate measure of Hsp70-depedence, as it leads to direct ubiquitination by CHIP independent of Hsp70. Additionally, a small amount of Hsp70 has been shown to copurify with many of the purified proteins used in our studies, including nNOS (Bender et al., 1999); thus, a condition without added Hsp70 is not a true Hsp70-free condition. To overcome this experimental limitation, we used the Hsp70 cochaperone BAG-1M and the small molecule activator of Hsp70 JG-98 to probe the dependence of nNOS ubiquitination on Hsp70 function. Hsp70 function is driven by a conformational change resulting from the hydrolysis of ATP to ADP,
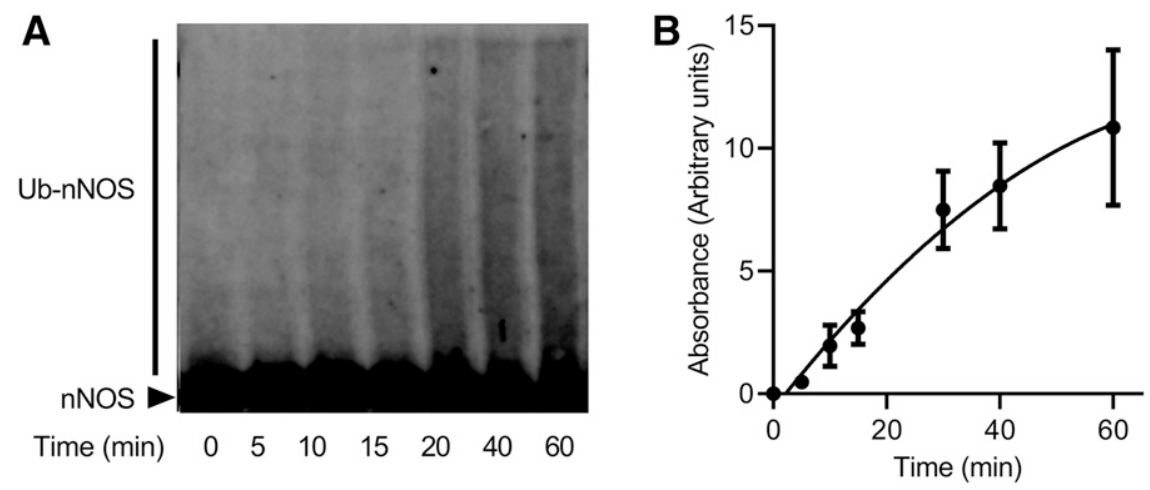

Fig. 1. Measurement of nNOS ubiquitination by Western blot. As described in Materials and Methods, FLAG-tagged apo-nNOS was incubated with a purified protein ubiquitination reaction mixture for increasing amounts of time. Resulting ubiquitinnNOS conjugates were visualized by Western blot utilizing an anti-nNOS antibody (A), and higher molecular weight mono- and poly-ubiquitin-nNOS conjugates were quantified (B). $(n=3)$ Values are reported as means \pm S.D. 


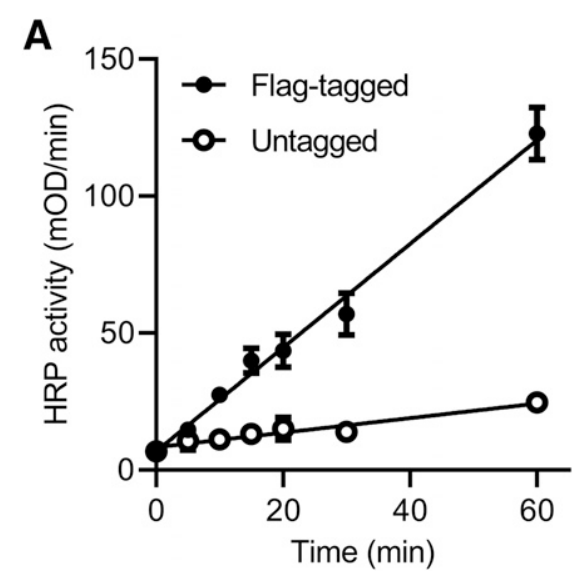

B

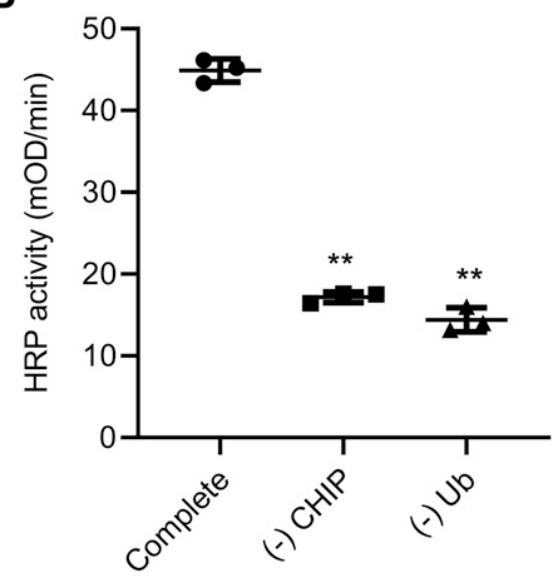

Fig. 2. Development of an ELISA to measure CHIP-dependent ubiquitination of FLAG-tagged nNOS. As described in Materials and Methods, FLAG-tagged apo-nNOS was incubated in a purified protein ubiquitination reaction mixture and immobilized on an anti-FLAG 96-well plate. The plate was probed with an anti-ubiquitin antibody conjugated to HRP, and HRP activity was quantified. (A) FLAG-tagged and untagged aponNOS were compared to assess the nNOS-dependence of the signal. FLAG-tagged (closed circles) and untagged (open circles) apo-nNOS were incubated for increasing amounts of time with the ubiquitination reaction mixture $(n=4)$. (B) To evaluate the dependence of the signal on CHIP-mediated ubiquitination, FLAG-tagged apo-nNOS was incubated for 15 minutes with the ubiquitination reaction mixture and mixtures lacking CHIP or ubiquitin (Ub) $(n=3)$. A repeated-measures one-way ANOVA followed by Dunnett's multiple comparisons test was used. Values represent means \pm S.D.; $* * P<0.01$.

and the ADP-bound conformation of Hsp70 has high affinity for client proteins (Zuiderweg et al., 2017). BAG1-M can act as a nucleotide exchange factor, promoting the release of ADP from Hsp70 (Bracher and Verghese, 2015). Although BAG1-M plays many roles in vivo (Rujano et al., 2007), in vitro BAG1-M has been shown to cause the dissociation of Hsp70 from client proteins (Rauch and Gestwicki, 2014). As shown in Fig. 3A, the ubiquitination reaction mixture was treated with BAG1-M. The addition of BAG1-M abolished apo-nNOS ubiquitination. The small molecule JG-98 is thought to stabilize Hsp70 in an ADP-like state and has been shown to antagonize the effect of BAG proteins (Li et al., 2015). Treatment of the mixture with JG-98 (closed circles) caused a 3-fold concentration-dependent increase in nNOS ubiquitination (Fig. 3B). Treatment of the ubiquitination reaction mixture with the JG-258 (open circles), an inactive analog of JG-98, had no effect on nNOS ubiquitination (Fig. 3B). These findings demonstrate that the
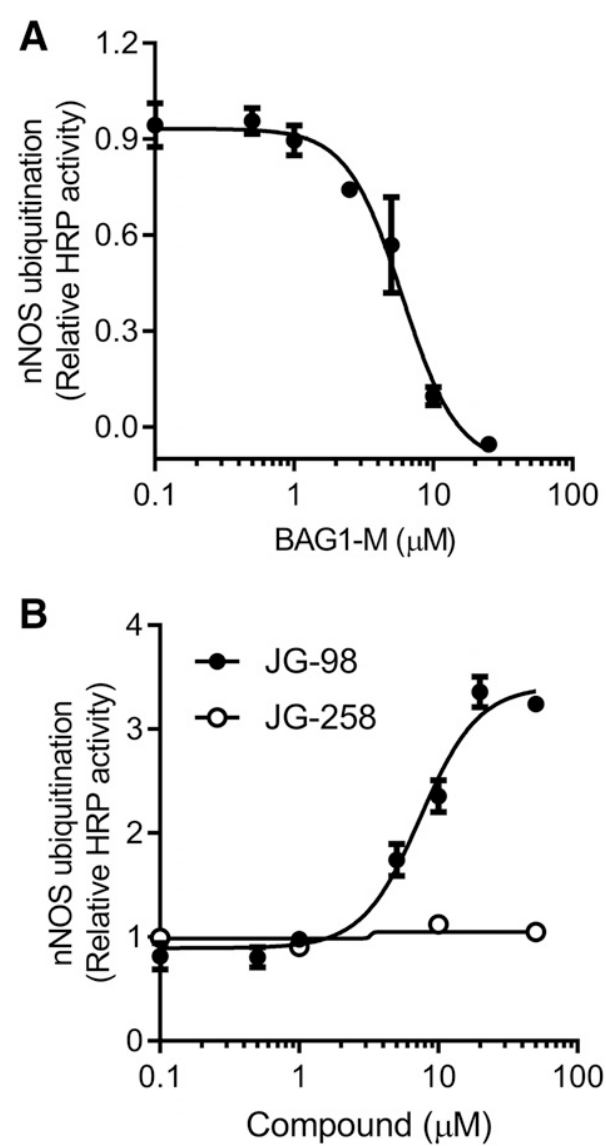

Fig. 3. Effect of Hsp70 modulators on nNOS ubiquitination. FLAG-tagged apo-nNOS was incubated with the purified protein ubiquitination reaction mixture for 15 minutes in the presence of increasing concentrations of the Hsp70 cochaperone BAG1-M $(n=3)(\mathrm{A})$ or the small molecule modulator of Hsp70 JG-98 (closed circles) and the structurally similar inactive analog JG-258 (open circles) $(n=3)$ (B).Values are reported as relative to untreated apo-nNOS and represent means \pm S.D.

nNOS ubiquitination observed in this system is dependent on Hsp70 function.

Hsp70 Preferentially Ubiquitinates Dysfunctional nNOS. We have demonstrated that alteration of the substratebinding cleft by autoinactivation and $\mathrm{BH}_{4}$ depletion triggers the ubiquitination and proteasomal degradation of nNOS (Bender et al., 2000a; Dunbar et al., 2004; Kamada et al., 2005), and stabilization of the substrate-binding cleft by slowly reversible inhibitors protects nNOS from degradation (Peng et al., 2012). As shown in Fig. 4, we used well established conditions and pharmacological tools to manipulate the substrate-binding cleft of nNOS to determine if the Hsp70:CHIP complex preferentially ubiquitinates dysfunctional nNOS.

Autoinactivation increases the ubiquitination of $\mathrm{nNOS}$ in cells, and stabilization of the substrate-binding cleft by L-NNA has been shown to block this effect (Peng et al., 2012). Studies in vitro have demonstrated that incubation of holo-nNOS with NADPH and calmodulin, in the absence of substrate, causes autoinactivation through the generation of reactive oxygen species within the heme-containing substratebinding cleft (Gebhart et al., 2019). Under these previously established conditions, we investigated the effect of autoinactivation of nNOS ubiquitination (Fig. 4A). Flag-tagged apo-nNOS or holo-nNOS was subjected to 30 minutes of 
A

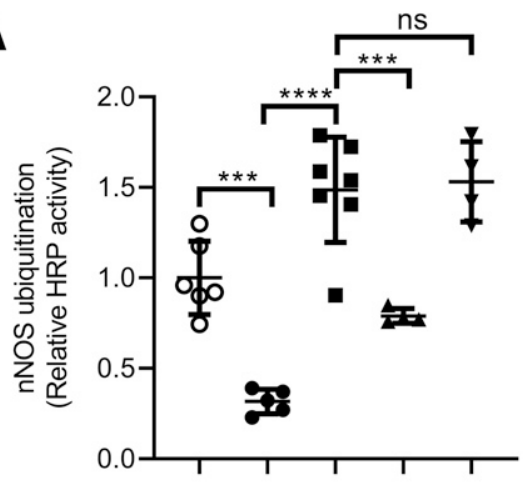

$\begin{array}{rccccc}\text { Apo-nNOS } & + & - & - & - & - \\ \text { Holo-nNOS } & - & + & + & + & + \\ \text { NADPH \& CaM } & - & - & + & + & + \\ \text { L-NNA } & - & - & - & + & - \\ \text { D-NNA } & - & - & - & - & +\end{array}$
B

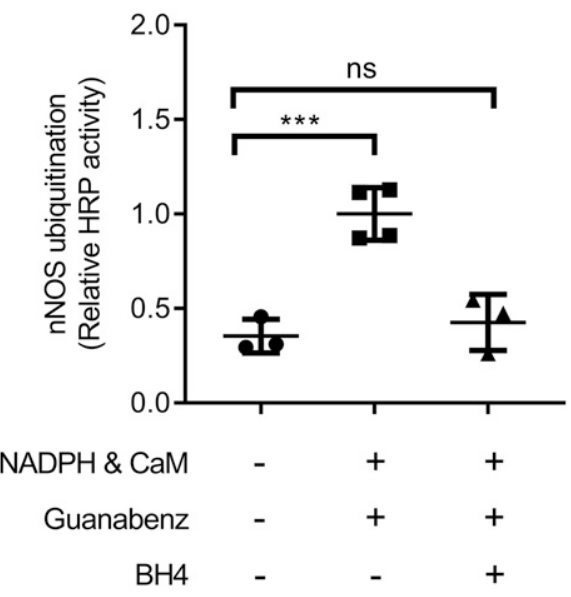

Fig. 4. Effect of substrate-binding cleft alteration on Hsp70-dependent ubiquitination of holo-nNOS. To investigate the effect of substrate-binding cleft alteration on $\mathrm{nNOS}$ ubiquitination, preincubations promoting autoinactivation and $\mathrm{BH}_{4}$ depletion of holo-nNOS were performed. FLAG-tagged nNOS was preincubated for 30 minutes at $30^{\circ} \mathrm{C}$ and then incubated with the purified protein in vitro ubiquitination mixture for an additional 30 minutes. (A) To investigate the effect of autoinactivation on nNOS ubiquitination, FLAG-tagged holo-nNOS was incubated without and with NADPH and calmodulin. FLAG-tagged apo-nNOS incubated without NADPH and calmodulin was used as a positive control. To probe the effect of cleft stabilization, FLAG-tagged holo-nNOS was preincubated with NADPH and calmodulin in the presence of L-NNA or D-NNA. Ordinary one-way ANOVA followed by Tukey's multiple comparisons test was used $(n=4-6)$. (B) The effect of $\mathrm{BH}_{4}$ depletion was investigated by preincubating holo-nNOS with the mechanism-based inactivator guanabenz, NADPH, and calmodulin with and without $\mathrm{BH}_{4}$. Ordinary one-way ANOVA followed by Dunnett's multiple comparisons test was used $(n=3$ to 4). Values are reported as relative to untreated apo-nNOS and represent means \pm S.D.; $* * * P<0.001 ; * * * * P<0.0001$. ns, not significant.

preincubation at $30^{\circ} \mathrm{C}$; in the case of holo-nNOS, this preincubation was with and without NADPH and calmodulin. In the absence of NADPH and calmodulin, the ubiquitination of apo-nNOS was significantly greater than holo-nNOS. This finding is consistent with studies in cell-based systems that demonstrate depletion of heme from the substrate-binding cleft of nNOS significantly increases ubiquitination (Dunbar et al., 2004). Preincubation with NADPH and calmodulin significantly increased the ubiquitination of holo-nNOS. To determine if this increase in ubiquitination reflects changes within the substrate-binding cleft, holo-nNOS was preincubated with NADPH and calmodulin in the presence of L-NNA (Fig. 4A). Treatment with L-NNA blocked the effect of NADPH and calmodulin on the ubiquitination of holonNOS. Treatment with the inactivate stereoisomer D-NNA had no effect on the increase in ubiquitination caused by NADPH and calmodulin. These findings demonstrate that alteration of the substrate-binding cleft through autoinactivation increases ubiquitination of holo-nNOS by the Hsp70:CHIP complex.

As another way to alter the substrate-binding cleft, we used the metabolism-based inactivator guanabenz. In a process that requires NADPH and calmodulin, guanabenz causes the oxidation and ensuing loss of $\mathrm{BH}_{4}$ from the substrate-binding cleft of nNOS (Noguchi et al., 2000; Dunbar et al., 2006), leading to rapid ubiquitination and proteasomal degradation in vivo (Nakatsuka et al., 1998). As shown in Fig. 4B, FLAG-tagged holo-nNOS was preincubated with or without NADPH, calmodulin, and $100 \mu \mathrm{M}$ guanabenz. Preincubation with NADPH, calmodulin, and guanabenz significantly increased ubiquitination of holo-nNOS. Additionally, cotreatment with $\mathrm{BH}_{4}$ abolished the effect of guanabenz (Fig. 4B). These findings demonstrate that depletion of $\mathrm{BH}_{4}$ from the substrate-binding cleft increases the ubiquitination of holo-nNOS by the Hsp70:CHIP-complex. These findings demonstrate that the minimal purified protein system for Hsp70-dependent ubiquitination successfully recapitulates the selective ubiquitination of dysfunctional nNOS that is observed in vivo.

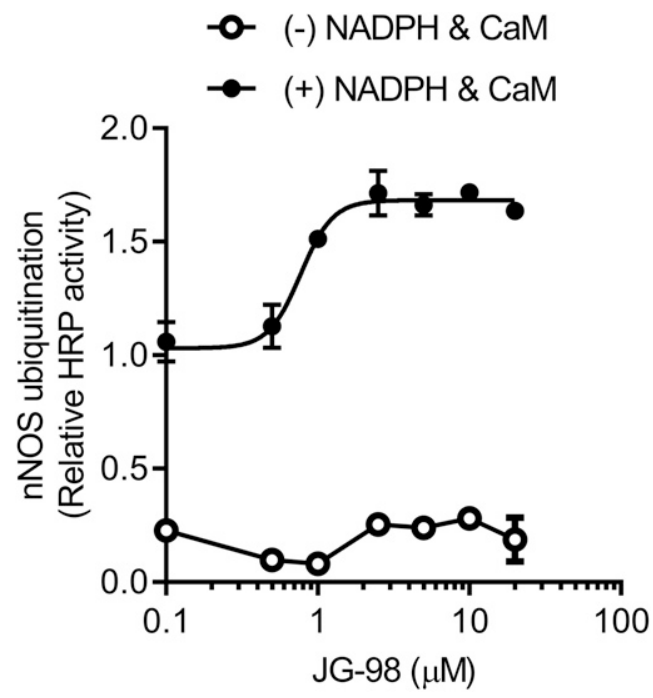

Fig. 5. Effect of pharmacological activation of Hsp70 on holo-nNOS ubiquitination. To determine if pharmacological activation alters the selectivity of Hsp70-dependent ubiquitination, FLAG-tagged holo-nNOS was preincubated with (closed circles) and without (open circles) NADPH and calmodulin (CaM). FLAG-tagged holo-nNOS was then transferred to the purified protein ubiquitination reaction mixture treated with increasing amounts of JG-98 and incubated for 15 minutes $(n=3)$. Values are reported as relative to untreated apo-nNOS and represent means \pm S.D. 
Pharmacological Activation of Hsp70 Maintains the Selectivity for Ubiquitination of Dysfunctional nNOS. We next sought to investigate whether the activation of Hsp70 would alter the selectivity of the ubiquitination such that native functional forms of nNOS would also be ubiquitinated. To address this, we assessed the effect of JG-98 on holo-nNOS as our representative of native protein compared with that of the autoinactivated holo-nNOS obtained by redox cycling with NADPH in the presence of calmodulin, as shown above in Fig. 4A. As shown in Fig. 5, JG-98 caused a concentrationdependent increase in the ubiquitination of autoinactivated holo-nNOS (closed circles) as would be expected from activation of Hsp70 shown earlier for apo-nNOS. Interestingly, the native holo-nNOS was unaffected by JG98 (open circles). These results demonstrate that activation of Hsp70 by JG98 does not abolish the selectivity of ubiquitination by the Hsp70:CHIP complex.

\section{Discussion}

In this study, we have shown that the Hsp70:CHIP complex preferentially ubiquitinates dysfunctional forms of nNOS over their native conformations, and activation of Hsp70 by the small molecule JG-98 selectively increases the ubiquitination of dysfunctional nNOS. These findings demonstrate Hsp70dependent ubiquitination is selective for dysfunctional nNOS, as experiments were performed under assay conditions optimized for Hsp70-dependent ubiquitination by the Hsp70: CHIP complex, free of direct ubiquitination by CHIP. Clearly, in this study we only investigated the selectivity of the Hsp70: CHIP complex; however, in cells, Hsp70 and Hsp90 work together as part of a multiprotein chaperone complex. It has been shown that Hsp70 facilitates the loading of Hsp90 onto client proteins and that Hsp90 stabilizes protein-folding clefts, protecting client proteins from degradation (Pratt et al., 2008). Thus, inhibition of Hsp90 increases the ubiquitination and degradation of these otherwise stabilized proteins. Conversely, this study suggests that activation of Hsp70 does not alter the specificity of ubiquitination and instead selectively enhances the ubiquitination of dysfunctional proteins. This finding is of therapeutic interest because activation of Hsp70 has been proposed as a strategy to increase the degradation of disease-causing client proteins (Pratt et al., 2015; Davis et al., 2020), and a concomitant increase in the degradation of native proteins would pose a potential liability.

Much of our understanding of the selectivity of Hsp70dependent ubiquitination comes from landmark studies on heat-denatured firefly luciferase. For example, it was demonstrated Hsp70 and CHIP facilitated the selective ubiquitination of heat-denatured firefly luciferase over the native form in vitro (Murata et al., 2001). In the current study, we chose nNOS as a model client protein because we have previously established that alteration of the substrate-binding cleft creates dysfunctional forms of nNOS, which are selectively targeted for ubiquitination and proteasomal degradation in cells and in vivo (Peng et al., 2012; Pratt et al., 2014). Therefore, utilizing well established methods to generate these dysfunctional forms in vitro allowed us to investigate the selectivity of Hsp70-dependent ubiquitination with naturally occurring non-native species. Guanabenz is a mechanism-based inactivator of nNOS that causes oxidation and eventual loss of $\mathrm{BH}_{4}$ (Bender et al., 2000a; Noguchi et al.,
2000; Dunbar et al., 2006), an essential cofactor bound to the substrate-binding cleft of nNOS, leading to rapid ubiquitination and proteasomal degradation of nNOS in vivo (Nakatsuka et al., 1998). Moreover, this effect is ameliorated when $\mathrm{BH}_{4}$ is administered (Dunbar et al., 2006). Additionally, depletion of heme from the substrate-binding cleft enhances nNOS ubiquitination in cell-based systems (Bender et al., 2000a; Dunbar et al., 2004). Consistent with these previous findings from cells and animals, in the current study, we observed that the Hsp70:CHIP complex selectively ubiquitinates heme- and $\mathrm{BH}_{4}$-depleted dysfunctional forms of $\mathrm{nNOS}$ in vitro. It is important to note that these dysfunctional forms of nNOS are not denatured, as they still maintain NADPH oxidase activity (Abu-Soud et al., 1994b; Gebhart et al., 2019) and represent forms that naturally occur in vivo. Interestingly, for all three NOS isoforms, depletion of $\mathrm{BH}_{4}$ leads to an uncoupled form of the enzyme that produces superoxide (Gebhart et al., 2019), contributing to oxidative stress in disease. For example, uncoupling of nNOS and the endothelial isoform endothelial nitric oxide synthase is thought to play a role in cardiovascular disease (Farah et al., 2018). Thus, selective degradation of this dysfunctional form may be therapeutically beneficial. Further studies utilizing relevant in vivo models will be required to confirm the significance of this study's in vitro findings.

The ELISA-based method developed in our current study allows for reproducible and quantitative measurement of Hsp70-dependent ubiquitination of nNOS in a 96-well format, offering a major advance over more laborious and timeintensive Western blotting-based approaches. Moreover, as discussed above, with the use of nNOS as a model client protein, this method provided a facile approach to test the effect of Hsp70 modulators on the selectivity of ubiquitination for different conformations of the client protein. In the current study, we investigated the effect of the rhodacyanine compound JG-98 and demonstrated, as proof of concept, that activation of Hsp70 can selectively enhance the ubiquitination of dysfunctional proteins. However, numerous small molecules targeting Hsp70 are under development for the treatment of various diseases ( $\mathrm{Li}$ et al., 2016; Gestwicki and Shao, 2019), and it is unknown if compounds with different mechanisms of action would abolish or maintain selectivity. Additionally, Hsp70 regulates numerous processes in addition to ubiquitination, including client protein folding and maturation; interference with these critical functions could also lead to toxicity. Thus, in combination with well established in vitro methods to measure other Hsp70-mediated functions, such as heme insertion into nNOS (Bender et al., 2000b) or glucocorticoid receptor steroid binding (Dittmar et al., 1996), the method described in this study may help in assessing the potential liability of compounds targeting Hsp70 for therapeutic use.

Both genetic and pharmacological activation of Hsp70 have been shown to enhance the ubiquitination and degradation of numerous disease-causing client proteins, including many prone to misfolding, making Hsp70 an attractive therapeutic target (Pratt et al., 2015; Davis et al., 2020). We have previously demonstrated that treatment with the Hsp70 cochaperone Hsp70-interacting protein (HIP), known to stabilize the ADP-bound state of Hsp70 and increase affinity for client protein, or the "HIP-like" rhodacyanine compound YM01, increases the ubiquitination of nNOS in cells (Wang et al., 2013). Moreover, treatment with HIP or YM-01 increased the 
clearance of the polyglutamine androgen receptor in cells and alleviated disease phenotype in a drosophila model of Kennedy's disease, a muscular and neurodegenerative disorder caused by the misfolding and aggregation of polyglutamine androgen receptor (Wang et al., 2013). As with nNOS, it has been demonstrated that the Hsp90 and Hsp70 machinery surveils protein-folding clefts of other client proteins, such as steroid receptors (Kaul et al., 2002) and tyrosine kinases (Citri et al., 2002; Xu et al., 2005), and selectively targets non-native forms for degradation (Pratt et al., 2015). Therefore, pharmacological activation of Hsp70 may provide a broad therapeutic approach to selectively dispose of a wide variety of diseasecausing client proteins.

\section{Acknowledgments}

Thank you to Jason Gestwicki from the University of California, San Francisco, for providing the small molecules JG-98 and JG-258.

\section{Authorship Contributions}

Participated in research design: Davis, Osawa.

Conducted experiments: Davis, McMyn.

Contributed new reagents or analytic tools: Davis, Lau, Morishima. Performed data analysis: Davis, McMyn.

Wrote or contributed to the writing of the manuscript: Davis, Osawa.

\section{References}

Abu-Soud HM, Feldman PL, Clark P, and Stuehr DJ (1994a) Electron transfer in the nitric-oxide synthases. Characterization of L-arginine analogs that block heme iron reduction. J Biol Chem 269:32318-32326.

Abu-Soud HM, Yoho LL, and Stuehr DJ (1994b) Calmodulin controls neuronal nitricoxide synthase by a dual mechanism. Activation of intra- and interdomain electron transfer. J Biol Chem 269:32047-32050.

Ballinger CA, Connell P, Wu Y, Hu Z, Thompson LJ, Yin LY, and Patterson C (1999) Identification of CHIP, a novel tetratricopeptide repeat-containing protein that interacts with heat shock proteins and negatively regulates chaperone functions. Mol Cell Biol 19:4535-4545.

Bender AT, Demady DR, and Osawa Y (2000a) Ubiquitination of neuronal nitricoxide synthase in vitro and in vivo. J Biol Chem 275:17407-17411.

Bender AT, Nakatsuka M, and Osawa Y (2000b) Heme insertion, assembly, and activation of apo-neuronal nitric-oxide synthase in vitro. $J$ Biol Chem 275: 26018-26023.

Bender AT, Silverstein AM, Demady DR, Kanelakis KC, Noguchi S, Pratt WB, and Osawa Y (1999) Neuronal nitric-oxide synthase is regulated by the Hsp90based chaperone system in vivo. J Biol Chem 274:1472-1478.

Bracher A and Verghese $J$ (2015) The nucleotide exchange factors of Hsp70 molecular chaperones. Front Mol Biosci 2:10.

Citri A, Alroy I, Lavi S, Rubin C, Xu W, Grammatikakis N, Patterson C, Neckers L, Fry DW, and Yarden Y (2002) Drug-induced ubiquitylation and degradation of ErbB receptor tyrosine kinases: implications for cancer therapy. EMBO $J \mathbf{2 1}$ : 2407-2417.

Davis AK, Pratt WB, Lieberman AP, and Osawa Y (2020) Targeting Hsp70 facilitated protein quality control for treatment of polyglutamine diseases. Cell Mol Life Sci 77:977-996.

Dittmar KD, Hutchison KA, Owens-Grillo JK, and Pratt WB (1996) Reconstitution of the steroid receptor.hsp90 heterocomplex assembly system of rabbit reticulocyte lysate. J Biol Chem 271:12833-12839.

Dunbar AY, Jenkins GJ, Jianmongkol S, Nakatsuka M, Lowe ER, Lau M, and Osawa Y (2006) Tetrahydrobiopterin protects against guanabenz-mediated inhibition of neuronal nitric-oxide synthase in vitro and in vivo. Drug Metab Dispos 34: 1448-1456.

Dunbar AY, Kamada Y, Jenkins GJ, Lowe ER, Billecke SS, and Osawa Y (2004) Ubiquitination and degradation of neuronal nitric-oxide synthase in vitro: dimer stabilization protects the enzyme from proteolysis. Mol Pharmacol 66:964-969.

Farah C, Michel LYM, and Balligand JL (2018) Nitric oxide signalling in cardiovascular health and disease. Nat Rev Cardiol 15:292-316.

Gässler CS, Wiederkehr T, Brehmer D, Bukau B, and Mayer MP (2001) Bag-1M accelerates nucleotide release for human $\mathrm{Hsc70}$ and $\mathrm{Hsp70}$ and can act concentration-dependent as positive and negative cofactor. J Biol Chem 276 $32538-32544$.
Gebhart V, Reiß K, Kollau A, Mayer B, and Gorren ACF (2019) Site and mechanism of uncoupling of nitric-oxide synthase: uncoupling by monomerization and other misconceptions. Nitric Oxide 89:14-21.

Gestwicki JE and Shao H (2019) Inhibitors and chemical probes for molecular chaperone networks. J Biol Chem 294:2151-2161.

Isaacs JS, Xu W, and Neckers L (2003) Heat shock protein 90 as a molecular target for cancer therapeutics. Cancer Cell 3:213-217.

Kamada Y, Jenkins GJ, Lau M, Dunbar AY, Lowe ER, and Osawa Y (2005) Tetrahydrobiopterin depletion and ubiquitylation of neuronal nitric oxide synthase. Brain Res Mol Brain Res 142:19-27.

Kaul S, Murphy PJ, Chen J, Brown L, Pratt WB, and Simons SS Jr. (2002) Mutations at positions 547-553 of rat glucocorticoid receptors reveal that hsp90 binding requires the presence, but not defined composition, of a seven-amino acid sequence at the amino terminus of the ligand binding domain. $J$ Biol Chem 277: 36223-36232.

Li X, Colvin T, Rauch JN, Acosta-Alvear D, Kampmann M, Dunyak B, Hann B, Aftab BT, Murnane M, Cho M, et al. (2015) Validation of the Hsp70-Bag3 protein-protein interaction as a potential therapeutic target in cancer. Mol Cancer Ther 14: $642-648$.

Li X, Shao H, Taylor IR, and Gestwicki JE (2016) Targeting allosteric control mechanisms in heat shock protein 70 (Hsp70). Curr Top Med Chem 16:2729-2740.

Li X, Srinivasan SR, Connarn J, Ahmad A, Young ZT, Kabza AM, Zuiderweg ERP, Sun D, and Gestwicki JE (2013) Analogues of the allosteric heat shock protein 70 (Hsp70) inhibitor, MKT-077, as anti-cancer agents. ACS Med Chem Lett 4: 1042-1047.

McDonough H and Patterson C (2003) CHIP: a link between the chaperone and proteasome systems. Cell Stress Chaperones 8:303-308.

Morishima Y, Zhang H, Lau M, and Osawa Y (2016) Improved method for assembly of hemeprotein neuronal NO-synthase heterodimers. Anal Biochem 511:24-26.

Murata S, Minami Y, Minami M, Chiba T, and Tanaka K (2001) CHIP is a chaperonedependent E3 ligase that ubiquitylates unfolded protein. EMBO Rep 2:1133-1138.

Nakatsuka M, Nakatsuka K, and Osawa Y (1998) Metabolism-based inactivation of penile nitric oxide synthase activity by guanabenz. Drug Metab Dispos 26:497-501.

Noguchi S, Jianmongkol S, Bender AT, Kamada Y, Demady DR, and Osawa Y (2000) Guanabenz-mediated inactivation and enhanced proteolytic degradation of neuronal nitric-oxide synthase. J Biol Chem 275:2376-2380.

Peng H-M, Morishima Y, Pratt WB, and Osawa Y (2012) Modulation of heme/substrate binding cleft of neuronal nitric-oxide synthase (nNOS) regulates binding of Hsp90 and Hsp70 proteins and nNOS ubiquitination. $J$ Biol Chem 287:1556-1565.

Pratt WB, Gestwicki JE, Osawa Y, and Lieberman AP (2015) Targeting Hsp90/ Hsp70-based protein quality control for treatment of adult onset neurodegenerative diseases. Annu Rev Pharmacol Toxicol 55:353-371.

Pratt WB, Morishima Y, Gestwicki JE, Lieberman AP, and Osawa Y (2014) A model in which heat shock protein 90 targets protein-folding clefts: rationale for a new approach to neuroprotective treatment of protein folding diseases. Exp Biol Med (Maywood) 239:1405-1413.

Pratt WB, Morishima Y, and Osawa Y (2008) The Hsp90 chaperone machinery regulates signaling by modulating ligand binding clefts. $J$ Biol Chem 283: $22885-22889$.

Rauch JN and Gestwicki JE (2014) Binding of human nucleotide exchange factors to heat shock protein 70 (Hsp70) generates functionally distinct complexes in vitro. $J$ Biol Chem 289:1402-1414.

Ravalin M, Theofilas P, Basu K, Opoku-Nsiah KA, Assimon VA, Medina-Cleghorn D, Chen YF, Bohn MF, Arkin M, Grinberg LT, et al. (2019) Specificity for latent C termini links the E3 ubiquitin ligase CHIP to caspases. Nat Chem Biol 15:786-794.

Ren HY, Patterson C, Cyr DM, and Rosser MF (2011) Reconstitution of CHIP E3 ubiquitin ligase activity. Methods Mol Biol 787:93-103.

Rujano MA, Kampinga HH, and Salomons FA (2007) Modulation of polyglutamine inclusion formation by the Hsp70 chaperone machine. Exp Cell Res 313:3568-3578.

Sanchez J, Carter TR, Cohen MS, and Blagg BS (2020) Old and new approaches to target the Hsp90 chaperone. Curr Cancer Drug Targets 20:253-270.

Stuart JK, Myszka DG, Joss L, Mitchell RS, McDonald SM, Xie Z, Takayama S, Reed JC, and Ely KR (1998) Characterization of interactions between the anti-apoptotic protein BAG-1 and Hsc70 molecular chaperones. J Biol Chem 273:22506-22514.

Wang AM, Miyata Y, Klinedinst S, Peng H-M, Chua JP, Komiyama T, Li X, Morishima Y, Merry DE, Pratt WB, et al. (2013) Activation of Hsp70 reduces neurotoxicity by promoting polyglutamine protein degradation. Nat Chem Biol 9: $112-118$.

Xu W, Yuan X, Xiang Z, Mimnaugh E, Marcu M, and Neckers L (2005) Surface charge and hydrophobicity determine ErbB2 binding to the Hsp90 chaperone complex. Nat Struct Mol Biol 12:120-126.

You J and Pickart CM (2001) A HECT domain E3 enzyme assembles novel polyubiquitin chains. J Biol Chem 276:19871-19878.

Zuiderweg ERP, Hightower LE, and Gestwicki JE (2017) The remarkable multivalency of the Hsp70 chaperones. Cell Stress Chaperones 22:173-189.

Address correspondence to: Yoichi Osawa, Department of Pharmacology, University of Michigan, 1150 W Medical Center Dr., Ann Arbor, MI 48109. E-mail: osawa@umich.edu 